\title{
Serum Biomarkers in Postoperative Delirium after Esophagectomy
}

Azhar Dalal ${ }^{1}$, Sikandar Khan ${ }^{2,3}$, Heidi Lindroth ${ }^{3,4}$, Babar Khan ${ }^{2,3}$

${ }^{1}$ Indiana University School of Medicine, ${ }^{2}$ Division of Pulmonary, Critical Care, Sleep and

Occupational Medicine, Department of Medicine, Indiana University School of Medicine, Indianapolis, IN, ${ }^{3} \mathrm{IU}$ Center of Aging Research, Regenstrief Institute, Indianapolis, IN, ${ }^{4}$ Indiana University School of Nursing, Indianapolis, Indiana

Background: The pathophysiology of postoperative delirium in patients undergoing esophagectomy is not well understood but has been hypothesized to involve neuroinflammation. Inflammatory biomarkers may therefore be associated with delirium incidence and severity. We conducted this study to measure the association between serum inflammatory biomarkers and postoperative delirium and delirium severity.

Methods: Blood samples were obtained from patients enrolled in a randomized double-blind placebo-controlled trial undergoing elective esophagectomy at Indiana University Health University Hospital. S100 calcium-binding protein B, C-Reactive Protein (CRP), interleukins 8, 10, tumor necrosis factor-alpha, and insulin like growth factor 1 (IGF1) were analyzed at preoperative, and postoperative days 1 and 3 timepoints. Delirium status and severity were assessed using the Confusion Assessment Method for Intensive Care Unit and Delirium Rating Scale (DRS-R-98), respectively.

Results: Samples from 71 patients were included. Higher median CRP values were associated with delirium incidence at post-operative day 1 (no delirium: 22.7 IQR:2.2, 42.7; delirium: 35.4 IQR:25.0, 50.0, p=0.031) but not at post-operative day 3. Median CRP S100B serum values were correlated with mean delirium severity $(-0.289, p=0.020)$. IGF-1 serum values were associated with delirium severity on post-operative day $1(0.270, p=0.040)$.

Conclusion: Postoperative delirium incidence was associated with higher median CRP values at POD1. The findings of this study provide evidence for the early measurement of postoperative serum biomarkers to ascertain likely incidence and severity of delirium. 